\title{
Sensitivity to event timing in regular and irregular sequences: Influences of musical skill
}

\author{
WILLIAM YEE, SUSAN HOLLERAN, and MARI RIESS JONES \\ Ohio State University, Columbus, Ohio
}

\begin{abstract}
In two experiments, the performance of listeners with different amounts of musical training (high skill, low skill) was examined in a two-alternative forced choice time-detection task involving simple five-cycle acoustic sequences. In each of a series of trials, all listeners determined which of two pattern cycles contained a small time change. Sequence context was also varied (regular vs. irregular timing). In Experiment 1, in which context was manipulated as a between-subjects variable, highskill listeners performed significantly better than low-skill listeners only with regular patterns. In Experiment 2 , in which context was manipulated as a within-subjects variable, the only significant source of variance was pattern context: All listeners were better at detecting time changes in regular than in irregular patterns. The results are considered in light of several hypotheses, including the expectancy/contrast model (Jones \& Boltz, 1989).
\end{abstract}

In this research we considered whether musical skill influenced listeners' detection of small timing changes in simple acoustic tone patterns. The literature had yielded mixed reports on skill differences. For example, Essens and Povel (1985) found no differences between musicians and nonmusicians in reproductions of time intervals within metrical (regular) and nonmetrical (irregular) sequences. Others, using a time-change detection task, report a superiority for musicians (Jones, Jagacinski, Yee, Floyd, \& Klapp, in press; see also Sloboda, 1983). While the tasks involved differed (reproduction vs. detection), it is unlikely that this difference explains the discrepancy: Bharucha and Pryor (1986), like Jones et al. (in press), used a time-change detection task, but nonetheless found no skill differences. Another possibility relates to design differences associated with manipulations of sequence structure; repeated measures designs are common in investigations that report negligible skill differences. The present research used both withinand between-subjects designs to control the number and kind of sequences listeners experience. We used a twoalternative forced choice (2AFC) procedure in an attempt to understand the effects of skill and pattern context on listeners' ability to detect small time changes within these sequences.

Using isochronous monotone sequences, benchmark temporal difference limens associated with timedetection/discrimination tasks have been reported in the neighborhood of $6 \%$ over a range of interonset interval

\footnotetext{
The authors gratefully acknowledge the contributions of Terry Lo for his assistance in experimental design and data collection. They are also indebted to Steve Handel and two anonymous reviewers for comments on an earlier version of this manuscript. Requests for reprints should be directed to M. R. Jones, Department of Psychology, 137 Townshend Hall, Ohio State University, Columbus, OH 43210.
}

(IOI) values (e.g., Halpern \& Darwin, 1984; Hirsh, Monahan, Grant, \& Singh, 1990; Van Noorden, 1975). In these sequences, if the time interval, $T$, of the Weber fraction, (JND) $/ T$, is taken as the constant IOI (where JND refers to a just noticeable time difference), then the above findings are consistent with Weber's law. Because these thresholds agree (roughly) with earlier findings based on isolated pairs of time intervals (e.g., Abel, 1972; Allan, 1979; Creelman, 1962; Getty, 1975; Small \& Campbell, 1962), Monahan and Hirsh (1990) concluded that "temporal interval discrimination for tonal onsets in a sequence of tones was similar to older results for the discrimination of single temporal intervals" (p. 227). However, there is also evidence that in various nonisochronous sequences, contextual relationships related to frequency and timing properties of sequence elements influence listeners' relative threshold for discriminating a time change (Bharucha \& Pryor, 1986; Hirsh et al., 1990; Jones et al., in press; Kidd \& Watson, 1990; Monahan \& Hirsh, 1990; Van Noorden, 1975). Accordingly, Monahan and Hirsh (1990) recently proposed a variant of Weber's law, the average Weber model, to describe rhythmic context effects observed in sequences where IOIs varied with corresponding changes in difference limens. Here $T$ is no longer based on the average (or fixed) IOI within an entire sequence but rather on the average of the two IOIs neighboring a time change $(\Delta t)$ within a sequence, in other words, on a local temporal context.

In the present research, we also assumed that detection of a small time change in a sequence was some function of the ratio of $\Delta t$ to a referent time span, $T$. However, we considered the possibility that this referent might be systematically influenced by training and by other, nonlocal, aspects of context. We asked whether trained listeners were better than untrained listeners at detecting a near-threshold time change in certain pattern 
contexts, and if so, why? If trained listeners were superior, this could be due to one (or both) of two factors related, respectively, to the numerator and denominator of a Weber fraction: (1) Skilled listeners, regardless of pattern context, may have greater temporal acuity; for the same $T$ value, they are sensitive to smaller absolute values of $\Delta t$ than other listeners. Or (2) skilled and unskilled listeners may have equivalent absolute time thresholds (JNDs), but for the same $\Delta t$ skilled listeners can exploit pattern context to arrive at a smaller effective $T$ value. In either case, a given time change should seem more noticeable to the skilled listener.

A means of differentiating between these two factors involves holding constant local temporal context, namely that immediately surrounding a fixed to-bedetected time change, and manipulating global context. Global pattern context refers to temporal intervals within a pattern that are remote from the time change. Figure 1 shows two five-cycle sequences, one regular and one irregular, involving alternating loud and soft tones. Cycle periods are marked by onsets of the louder, accented sounds, whereas IOIs separate loud and soft sounds. In both sequences, local context involves the second and fourth cycles, which are termed test cycles, because they may contain a time change. Cycle duration and IOIs within each test cycle are identical in regular and irregular sequences. These sequences differ primarily in cycle durations and IOIs of surrounding intervals, namely in global pattern context. In regular sequences, global context consists of invariant cycle durations; in irregular sequences, this duration varies.

In both patterns of Figure 1, cycles and IOIs are $e x$ plicitly marked time intervals, meaning that their endpoints correspond to sounded elements. However, regular patterns differ from irregular ones in their potential for specifying implicit time intervals (see dashed lines in Figure 1a). An implicit (or implied) time interval is one whose beginning and/or ending is not indicated by a sounded element (e.g., tonal onset). Regular patterns mimic metrical musical sequences in that their global context implies other time spans, ones not explicitly marked by tone onsets. Note that in Figure la smaller time spans are implied by a subdivision of the IOIs. In these patterns, the subdivision consistent with the cycle/IOI ratio involves a base 2 time ratio. That is, in both regular and irregular sequences, the IOI is always half the duration of a cycle, and hence softer tone onsets always occur at cycle midpoints. However, the variability in cycle duration in irregular sequences, means that a stable referent period for subdividing is absent, whereas in regular sequences such a referent is present; hence, an implied metrical beat (i.e., in this case, within a duple meter) is clearly specified.

To examine how these contextual factors affect timechange detection, a fixed time change $( \pm \Delta t)$ in the form of a shift in the softer tone is introduced into one of the

a)

REGULAR SEQUENCE

\section{CYCLE}

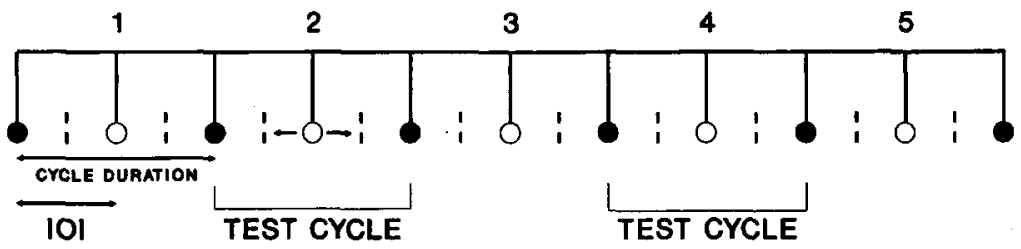

b)

IRREGULAR SEQUENCE

\section{CYCLE}

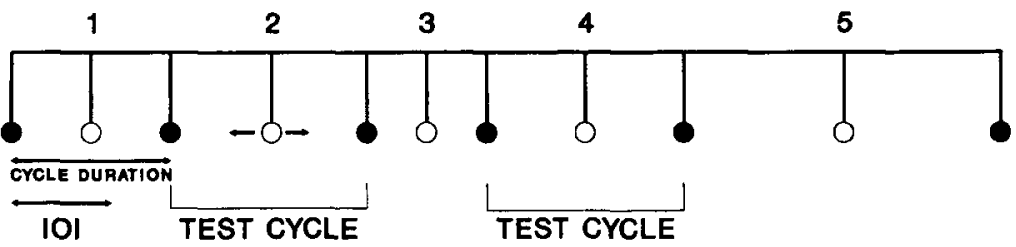

Figure 1. Examples of five-cycle sequences used in Experiments 1 and 2. Solid circles represent louder sounds marking cycle durations; open circles represent softer sounds which evenly subdivide each cycle. Panel a shows a regular sequence in which all cycles, including test cycles (cycles 2 and 4), have the same duration. Dashed vertical lines in the regular sequence suggest implied time intervals; depicted implied intervals reflect an even subdivision (base 2) of the interonset interval (IOI). Panel b shows an irregular sequence in which test cycles are identical in duration to those of regular sequences but durations of cycles 1,3 , and 5 vary randomly. 
two test cycles in each pattern. The duration of test cycles (i.e., cycles 2, 4) is constant, meaning that the average of the two IOIs neighboring a change is identical in both regular and irregular contexts. Primary differences concern global pattern context, namely durations of the three nontest cycles (i.e., cycles $1,3,5$ ). In the regular sequence, all durations of nontest cycles equal those of test cycles; in the irregular sequence, the duration of nontest cycles varies randomly about the same mean duration within a sequence (and over trials).

If musically trained listeners turn out to be superior to untrained listeners in time detection simply because they have greater acuity, then manipulations of global pattern context should not undermine their superiority. An acuity hypothesis posits that if a pattern's modal (or mean) IOI provides a referent $T$, then extended training will reduce the listener's temporal JND. In effect, the functional numerator of the Weber fraction is reduced in listeners with more training. This is one interpretation of Kristofferson's (1976) finding that practice produced uniformly small temporal JNDs over a range of $T$ intervals (see also Thomas \& Brown, 1974). That is, skill, but not pattern context, should affect performance.

Alternatively, global context may directly influence performance. In addition, effects of context may be more evident with skilled listeners who can "make the most" of pattern structure, perhaps by extracting an especially effective temporal reference frame, $T$. A null hypothesis along these lines is based on the average Weber model of Monahan and Hirsh (1990), which focuses on local temporal context, namely test cycle IOIs. Because averages of test cycle IOIs (mean $=800 \mathrm{msec}$ ) are identical for regular and irregular sequences, if these are the primary determinant of listeners' $T$, then global context should have no effect on performance.

Povel's clock model suggests how global aspects of pattern context may determine a $T$ value. This model rests on the assumption that regular sequences are temporally coded via a grid provided by a "best fitting" clock (Essens, 1986; Essens \& Povel, 1985; Povel \& Essens, 1985). The grid interval typically corresponds to the smallest explicit time interval that is regularly marked by a pattern's accented tones. ${ }^{1}$ Irregular sequences, however, are encoded as figural groupings and do not include temporal interval information. Assuming the best-fitting grid provides a determinate $T$, this model predicts a main effect of pattern context: Time detection will be more accurate with regular than with irregular sequences.

Finally, two other hypotheses also address global context by assuming that listeners use context to arrive at an estimated difference, or temporal contrast, between an expected time interval, ET, and an explicitly marked observed time interval, OT. Theoretical differences concern ET, the expected time span which underlies functional $T$ values. Kristofferson's (1977) real-time internal criterion model maintains that ET is an internal criterial time span which corresponds to the most frequently oc- curring, explicitly marked, time interval in an experimental session. Given a modal IOI for a session, then, estimates for $T$ are fixed and time discrimination depends on the range of IOIs within the session. As IOI range increases, time discrimination performance should decrease. Thus, in a between-subjects design, this model also predicts a main effect of pattern context: Listeners encountering only irregular sequences should perform more poorly than those encountering only regular sequences (Allan \& Kristofferson, 1974).

The second contrast hypothesis stems from the dynamic attending approach (Jones, 1976; Jones \& Boltz, 1989). The expectancy/contrast model predicts that regular pattern contexts are more likely than irregular ones to entrain attending and induce a future-oriented attending mode, a mode in which skilled listeners are more adept at using global pattern relationships. Regular pattern relationships promote anticipatory attending and expectancies, ET, based on time spans that are consistent with unfolding temporal regularities within a sequence. In particular, temporally invariant relationships pertaining to the cycle/IOI time ratio and cycle duration are proposed to influence listeners' expectancies in favor of certain time spans, ones consistent with IOIs and their multiples/subdivisions. Listeners with more musical training are assumed to be more capable of exploiting these relational properties. In fact, listeners who can abstract relevant time relations (e.g., time ratios) from a pattern's context can iteratively subdivide explicitly marked time spans to yield ET intervals that correspond to smaller, implied time values of $T$ will have an advantage. In principle, any ET can supply a $T$ value, but in practice smaller ETs, corresponding to implied time intervals, should help performance when listeners must detect small time changes. To explain this, we assume that time-detection performance depends on expectancy violation or a temporal contrast: OT - ET, which corresponds to the time difference between a tone's onset time and its expected time. Contrast amounts to a listener's estimate of the magnitude of an objective time change, $\Delta t$; for simplicity, $\Delta t^{\prime}$ hereafter denotes a temporal contrast. ${ }^{2}$ We assume that listeners' timedetection performance is some monotonic function of $\Delta t^{\prime} / \mathrm{ET}$ (Jones, Boltz, \& Klein, 1993). Furthermore, this model predicts an interaction of pattern context with skill level in that when provided with regular sequences, skilled listeners are more likely than less skilled listeners to generate small, implied ET values and hence to perform better.

Analytic attending, a memory-oriented attending mode, involves attending over relatively brief time spans. It is more likely with irregular sequences in which attentional entrainments are limited and the generation of expected time spans are difficult. In these cases, the $T$ values available to listeners of both skill levels will be highly variable and performance correspondingly poor. In short, this model predicts that both skill and global pattern context should affect performance, with 
skilled listeners performing unusually well with regular sequences.

Two experiments were designed to evaluate these various hypotheses about determinants of $T$. In both, pattern context (regular vs. irregular) and skill level (high vs. low) were manipulated. In Experiment 1, different groups of listeners responded to the regular and irregular sequences. In Experiment 2, all listeners received both kinds of sequences within the same experimental session. Of special interest were experimental design considerations. Specifically, do some hypotheses fare better (or worse) when structure is manipulated as a between-subjects variable?

\section{EXPERIMENT 1}

Experiment 1 relied on a between-subjects design to test hypotheses outlined in the introduction. Listeners in each of two different skill groups (low, high) encountered only one type of sequence, regular or irregular. Previous research had relied heavily upon repeated measures designs in which listeners heard a variety of different sequences within a session. While the advantages of such designs in terms of reduced costs and error variance are well known, classic "carryover" effects are also a risk (e.g., Jones, Kidd, \& Wetzel, 1981; Kidd, Boltz, \& Jones, 1984).

Accordingly, in Experiment 1, the primary variation over trials for listeners receiving regular sequences involved location (test cycle 2 vs. 4) and type (advanced vs. delayed) of time change in a sequence. The aim here was to hold constant both cycle duration and temporal subdivisions by softer tones within the sequence. Similar variations occurred over trials, with listeners receiving irregular sequences. However, the aim here was to vary cycle duration over trials while continuing to hold constant the relative timing of softer tones within cycles.

Experiment 1 was divided into two parts: Experiment 1a and Experiment $1 \mathrm{~b}$. In the former, different groups of listeners detected the same small time change in regular and irregular sequences with an average IOI of $800 \mathrm{msec}$. In Experiment $1 \mathrm{~b}$, as an added control for assessing subdividing strategies, listeners of low musical skill were presented with the same time change embedded in regular sequences having an IOI of $400 \mathrm{msec}$. In both experiments, the to-be-detected time change was $\pm 60 \mathrm{msec}$.

\section{Method}

\section{Subjects}

One hundred and eighteen Ohio State University students participated in Experiments $1 \mathrm{a}$ and $1 \mathrm{~b}$ in return for either course credit or money (\$6). In Experiment 1a, 53 were assigned to each of two musical skill groups (high, low) on the basis of criteria outlined below. The remaining 12 subjects (low skill) served in Experiment $\mathrm{lb}$. The following skill criteria were used:

High musical skill. Subjects must have had at least 4 years of formal musical training on an instrument, with some of the train- ing having occurred within the previous 5 years; these subjects must have played a musical instrument for at least 10 hours in the previous month.

Low musical skill. Subjects must not have played any musical instrument in the previous month. They must not have had any more than 2 years of total musical training with no formal musical training on any instrument within the previous 5 years.

\section{Design}

In a $2 \times 2 \times 2$ factorial design, two levels of musical skill (high, low) were crossed with two pattern context conditions (regular, irregular) and two counterbalanced orders in Experiment la. A single regular sequence (IOI) was presented to low-skill listeners in Experiment $1 \mathrm{~b}$.

\section{Apparatus}

All test stimuli were programmed using version 5.0 of the MIDILAB software (Todd, Boltz, \& Jones, 1989) on an IBM-PCcompatible computer interfaced by a Roland MPU-401 MIDI processing unit to control a Yamaha TX81Z FM tone generator set to a sine-wave voice. Stimulus patterns were recorded (Dolby B) on a Nakamichi LX-3 two-head cassette deck using TDK SA-X Super Avilyn Extra High Resolution cassettes. Cassettes were played (Dolby B) from a Nakamichi 550 tape deck adjusted for SX tapes. The output was delivered through a Kenwood Model KA-5700 integrated stereo amplifier to subjects wearing AKG K-240 stereo headphones. Both recording and playback were low-pass filtered through a Rockland Model 432 Dual Hi/Lo filter set to a $6000-\mathrm{Hz}$ cutoff frequency.

\section{Stimuli/Conditions}

Experiment 1a. The basic pattern for all stimuli was a loud-soft (two-beat) sequence having a total duration of $1,600 \mathrm{msec}$. The softer tone onset occurred at the midpoint of the pattern. Both tones were set at a frequency of $294 \mathrm{~Hz}\left(\mathrm{D}_{4}\right)$ and a duration of $60 \mathrm{msec}$. A trial consisted of five successive presentations (cycles) of the two-beat pattern, with the total sequence lasting $8,000 \mathrm{msec}$. The louder tone $(80 \mathrm{~dB})$ in each cycle served as the initial beat opening each cycle; the softer tone $(76 \mathrm{~dB})$ was the second beat. This manipulation induced a sequence of strong-weak beats where the strong beat initiated each cycle and the weak beat subdivided the cycle into two equal IOIs.

In regular conditions, all softer tones subdivided a fixed cycle duration of $1,600 \mathrm{msec}$, yielding a constant sequence of IOIs $(800 \mathrm{msec})$. Thus, except for a change in the timing of either the second or fourth cycle (test cycles), all cycles had identical time properties. The time change, $\Delta t$, corresponded to an advance or delay of the softer tone onset by $60 \mathrm{msec}$.

In irregular conditions, the stimuli were identical to those used in regular conditions except for non-test-cycle durations (cycles 1 , 3, 5; see Figure 1). As in regular patterns, the softer tone in test cycles occurred $60 \mathrm{msec}$ earlier or later than the midpoint in one of the test cycles (cycles 2, 4); test cycles lasted $1,600 \mathrm{msec}$. Within a given trial, durations of cycles 1,3 , and 5 were randomly varied in 100 -msec units from 1,000 to $2,200 \mathrm{msec}$, with an average of $1,600 \mathrm{msec}$ (none of these cycles was ever $1,600 \mathrm{msec}$ ). However (as with regular sequences), the softer tone in all cycles of irregular sequences continued to occur at the midpoint of each cycle. Total sequence duration remained $8,000 \mathrm{msec}$.

Experiment 1b. The basic stimuli and time change $( \pm 60 \mathrm{msec})$ in the second experiment were identical in all respects to the regular sequences of Experiment 1a, with one exception: The total cycle duration $(1,600 \mathrm{msec})$, marked by louder tones, was further subdivided by the addition of two more soft tones (at 400 and $1,200 \mathrm{msec}$ within the cycle) to yield four smaller, explicitly marked, time periods of $400 \mathrm{msec}$. Thus, the IOI of these regular sequences became $400 \mathrm{msec}$. 


\section{Procedure}

The procedures in Experiments $1 \mathrm{a}$ and $1 \mathrm{~b}$ were identical. Each experimental session consisted of three phases: train, practice, and test. The train phase familiarized subjects with the task. Listeners heard four trials containing an obvious timing change: the critical soft tone (in Experiment 1a, the only soft tone; in Experiment $1 \mathrm{~b}$, the second soft tone) was omitted in either the 2nd or 4th cycle. In a $2 \mathrm{AFC}$ task, the subjects were given approximately $10 \mathrm{sec}$ to indicate which cycle (either 2 or 4 ) was missing a tone by circling a 2 or a 4 on response sheets, after which a brief tone $(10548 \mathrm{~Hz}$ at $60 \mathrm{~dB}$ ) sounded. At this time, feedback was provided.

In the practice phase, the subjects received eight trials, as in training. The only difference was that the to-be-detected change in one of the test cycles was now a $\pm 60 \mathrm{msec}$ deviation of the softer tone. Instructions, supplemented by a diagram, indicated where timing changes might occur. In regular conditions, the diagram showed five identical cycle durations, while in irregular conditions it indicated the variability of cycles 1,3 , and 5 .

The test phase consisted of 64 trials identical to those used in the practice trials. Irregular conditions used the 32 patterns based on different variations of cycle durations, each presented twice for a total of 64 test trials. Half of the trials involved changes in cycle 2 , half in cycle 4 . Within each of these divisions, half of the trials involved a $60-\mathrm{msec}$ advance and half involved a $60-\mathrm{msec}$ delay. These changes were ordered over 32 trials according to two different randomizations (set 1,2 ) with the constraint that four successive sequences of eight trials within each set contain two trials of each combination: $+60 \mathrm{msec}$, cycle $2 ;-60 \mathrm{msec}$, cycle 2 ; $+60 \mathrm{msec}$, cycle 4 ; and $-60 \mathrm{msec}$, cycle 4 . One counterbalance order was created by the order set 1 , set 2 ; the other by the order set 2 , set 1 .

At the end of the session, listeners responded to a brief questionnaire which asked, among other things: (1) if they had listened metrically, (2) if they had anticipated the time change by projecting attention forward in time, and (3) what their listening strategy had been.

\section{Results and Discussion}

Due to high variability from outliers in the proportion correct scores (PC), two scores (the highest and lowest) were excluded from the analysis for each of the eight groups; this trimming succeeded in reducing variability while minimally affecting the means (Tukey, 1977). Analyses of PC are based on an analysis of variance (ANOVA) collapsed over counterbalance order, which had no significant effects. Trimmed mean PC values for each of the four resulting conditions (context $\times$ skill) in Experiment 1a are shown in Figure 2 along with the trimmed mean PC for unskilled listeners in Experiment $\mathrm{lb}$ who received regular sequences with more subdivisions.

Listeners in Experiment 1a, where the average IOI is $800 \mathrm{msec}$, did not perform equivalently across the four conditions, as might be anticipated if all were relying on the same $T$ value. Instead, mean performance ranged from a low of $58 \%$ correct (low skill, irregular) to a high of $92 \%$ correct (high skill, regular). Only low-skill listeners responding to regular sequences turned in mean performance levels in the neighborhood of previously established threshold levels (i.e., in a $2 \mathrm{AFC}$ procedure, threshold PC $=.75$, and this is observed to occur near $\Delta t / T=.06$ ). If $T$ equals the average IOI, then $\Delta t / T$ is .075 and the mean PC should be slightly above .75 . However, this is true only for low-skill listeners in regular context conditions (where $\mathrm{PC}=.76$ ).

Contributing to observed performance differences was musical skill $\left[F(1,94)=24.881, M S_{\mathrm{e}}=.0123, p<\right.$ $.001]$; high-skill listeners were significantly more accu-

\section{Effect of Musical Skill in Regular and Irregular Conditions}

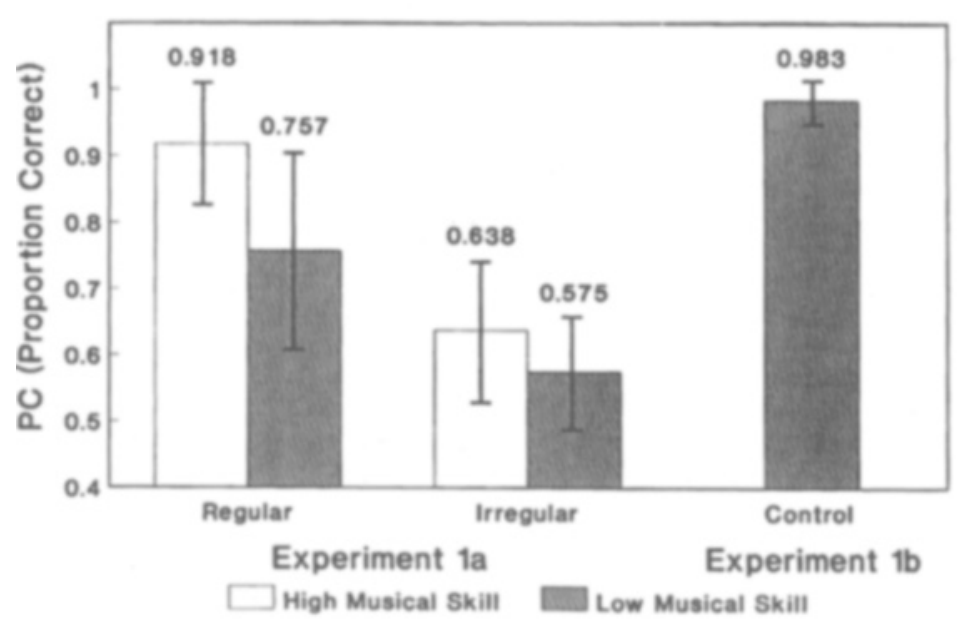

Figure 2. Mean proportion correct (PC) (also shown numerically) as a function of skill level and context in Experiments la and 1b. The data from Experiment 1b reflect performance of low-skill listeners with regular sequences having explicitly marked interonset intervals of $\mathbf{4 0 0} \mathbf{m s e c}$. Brackets indicate standard deviation per condition. 
rate $(M=.7807)$ than low-skill listeners $(M=.6682)$. In addition, all subjects were better at detecting timing changes in the regular sequences $(M=.8374)$ than in the irregular ones $(M=.6068)[F(1,94)=104.427$, $\left.M S_{\mathrm{e}}=.0125, p<.001\right]$, a finding that argues for the influence of pattern context on performance.

The most relevant finding concerning contextual influences on performance is a significant interaction of skill with context $\left[F(1,94)=4.684, M S_{\mathrm{e}}=.0125, p<\right.$ $.05]$, indicating that the advantages of skill are limited to the regular sequence condition. Listeners with high musical skill outperformed those with low musical skill only in regular pattern contexts; indeed, the former group performed at consistently high levels throughout the session [i.e., no significant improvement occurred with high-skill listeners over the eight trial blocks of regular sequences, $F(7,168)=1.05$, n.s.]. A set of Tukey $H S D$ post hoc comparisons revealed that differences due to context (regular vs. irregular) were significant for both skill groups $(p<.01)$. However, in regular sequences, the high-skill listeners were significantly better than the low-skill listeners $(p<.01)$; a reliable skill difference did not obtain with irregular sequences.

The data of Experiment $1 \mathrm{~b}$ aid in interpreting the skill $\times$ context interaction of Experiment 1a. One explanation of this effect posits that skilled listeners bring to the task a metrical listening skill which they can apply to regular sequences; when provided with a constant cycle duration, these listeners can generate an expected time span, ET, that is smaller than the explicitly marked 800msec IOI of Experiment 1a. For example, if their ET corresponds to a metrical beat, this roughly yields an implied time span of $800 / 2=400 \mathrm{msec}$. If $T \sim 400 \mathrm{msec}$, then skilled subjects should be able to detect a time change of less than half of $\Delta t=60 \mathrm{msec}$ at least $71 \%$ of the time (e.g., Halpern \& Darwin, 1984). The performance of low-skill listeners in Experiment $1 \mathrm{~b}$ with regular sequences having IOIs of $400 \mathrm{msec}$ is consistent with such an interpretation. These listeners averaged $\mathrm{PC}=.98$ in detecting the same time change when the 400-msec intervals were explicitly marked.

Questionnaire data from Experiment 1a, shown in Table 1 , also shed light on the skill $\times$ context interaction. Binomial tests indicate that in only two cases did listeners answer "Yes" significantly more than expected by chance $(p=.50)$; both cases involved the high-skill listeners who received regular sequences. Using a $z$ approximation to the binomial distribution, significantly more high-skill listeners reported hearing these patterns metrically rather than nonmetrically $(Z=3.8, p<.001)$ and significantly more of these listeners reported attending in an anticipatory mode $(Z=2.2, p<.025)$. Because similar trends are evident in both skill groups, chi-square analyses over the four conditions showed no significant distributional differences on either question.

A finer examination of responses to irregular patterns suggests that they varied greatly in difficulty as a function of various factors. No single factor appeared deci-
Table 1

Proportion of Listeners Reporting Metrical and Anticipatory Attending in Experiment 1

\begin{tabular}{cccccc}
\hline & \multicolumn{3}{c}{ Pattern Context } \\
\cline { 2 - 3 } \cline { 5 - 5 } Skill Level & \multicolumn{2}{c}{ Regular } & & \multicolumn{2}{c}{ Irregular } \\
\cline { 2 - 3 } \cline { 5 - 6 } & Metric? & Anticipate? & & Metric? & Anticipate? \\
\hline High & .88 & .76 & & .48 & .56 \\
Low & .60 & .64 & & .36 & .64 \\
\hline
\end{tabular}

Note-Entries are proportions of listeners responding "Yes," "Did you listen metrically?" (i.e., Metric?), and "Did you listen forward to anticipate a possible time change?" (i.e., Anticipate?)

sive. For some irregular sequences in which strings of IOIs surrounding a test cycle were similar, an embedded time change seemed to be more obvious, but in other cases higher accuracy was associated with the occurrence of two distinctly different cycle durations surrounding a test cycle. Similarly, postsession questionnaire responses from subjects receiving irregular sequences indicated that they engaged in a variety of listening strategies, with some claiming to successfully ignore the first and last cycles and to concentrate only on comparing cycles 2 and 4 . In some cases, high-skill listeners clearly outperformed low-skill ones in these irregular patterns, but the reasons for this were not evident, and on average skill differences were not reliable. Overall, there were indications that the most difficult sequences were ones which: (1) followed a test cycle with a cycle of a very different duration; and/or (2) placed the time change at a serial location that separated two strings of accidentally similar IOIs. ${ }^{3}$ To assess the extent to which similarity of IOIs determined performance, the range of IOIs/sequence in irregular conditions was correlated with mean PC, yielding an $r=-.103[t(31)=0.56$, $p>.10]$. This suggests that overall homogeneity of IOIs within a sequence, as indexed by range, does not account for performance differences among irregular sequences.

\section{EXPERIMENT 2}

The findings of Experiment 1 suggested that in the present task skill differences cannot be entirely attributed to differences in acuity; rather, listeners' responses to global aspects of sequence structure are important. Experiment 2 continued to evaluate hypotheses about context and time detection by expanding the notion of global context to include an entire experimental session.

Experiment 2 had several goals. First, we attempted to resolve discrepancies in the literature regarding skill differences. Unlike other investigations (e.g., Bharucha \& Pryor, 1986; Essens \& Povel, 1985), Experiment 1 demonstrated pronounced differences due to musical training, especially with regular sequences. To assess whether or not these effects are more likely to arise in between-subjects designs, Experiment 2 varied pattern context (regular vs. irregular) as a within-subjects variable. Here listeners encountered both regular and irreg- 
ular patterns of Experiment 1a. If previous failures to find skill differences reside in use of repeated measures designs, then Experiment 2 should replicate these null findings.

Another goal of Experiment 2 involved an assessment of the impact of cycle stability over time spans longer than a single pattern. Both Kristofferson's model and the expectancy/contrast model imply that variability of temporal structure within a session can influence performance. Kristofferson's model predicts that equating the range of IOIs encountered within a session for the two pattern contexts (regular and irregular) will eliminate effects due to the context variable. The expectancy/contrast model predicts that an invariant cycle period within a session promotes higher order attentional entrainments and abstraction of the base 2 time ratio. Thus, session variability should hamper skilled listener's generation of implied beats and eliminate the skill $\times$ context interaction.

\section{Method}

The method of Experiment 2 was identical to that of Experiment la, with the following exceptions:

\section{Subjects}

Thirty-six Ohio State University students volunteered to participate in the experiment in return for either course credit or money (\$6). Half possessed high musical skill and half possessed low musical skill based on the criteria described in the first experiment.

\section{Design}

In a $2 \times 2 \times 2$ mixed factorial design, two levels of musical skill (high, low) were crossed with two counterbalance orders. The pattern context variable (regular and irregular) was the single withinsubjects variable.

\section{Stimuli/Conditions}

Both the regular and irregular sequence trials described in Experiment la were randomly mixed, as opposed to being separate conditions as in Experiment la.

\section{Procedure}

The procedures were identical to those of Experiment 1, except that both regular and irregular sequence diagrams were shown during the instruction period.

During the test phase, 32 regular and 32 irregular trials were presented randomly with the constraint that there be no more than three consecutive trials of each structure type. Each half of the test phase contained an equal number of regular and irregular trials. The presentation order of the four types of to-be-detected changes, $\Delta t$ ( $+60 \mathrm{msec}$, cycle $2 ;-60 \mathrm{msec}$, cycle $2 ;+60 \mathrm{msec}$, cycle 4 ; and $-60 \mathrm{msec}$, cycle 4), were identical to the orders used in Experiment 1, dependent on whether the first or second counterbalance order was given. All 32 random irregular patterns generated in Experiment 1 were presented only once.

\section{Results and Discussion}

The mean PC for each of the resulting conditions (context $\times$ skill) are shown in Figure 3. An ANOVA based on PC scores, collapsed over counterbalance order (a nonsignificant variable), indicated that the context $X$ skill interaction observed in Experiment 1, disappeared in Experiment $2[F(1,34)=1.00]$. In addition, no significant differences were observed as a function of musical training $[F(1,34)=1.00]$. Skilled listeners did not perform significantly better than less skilled listeners in either pattern context. In fact, only context yielded reliable effects on performance. All subjects were significantly more accurate with regular sequences than with irregular ones $\left[F(1,34)=22.907, M S_{\mathrm{e}}=.0099, p<\right.$ $.001]$. Mean PC for the former was . 731 while for the latter it was .618 .

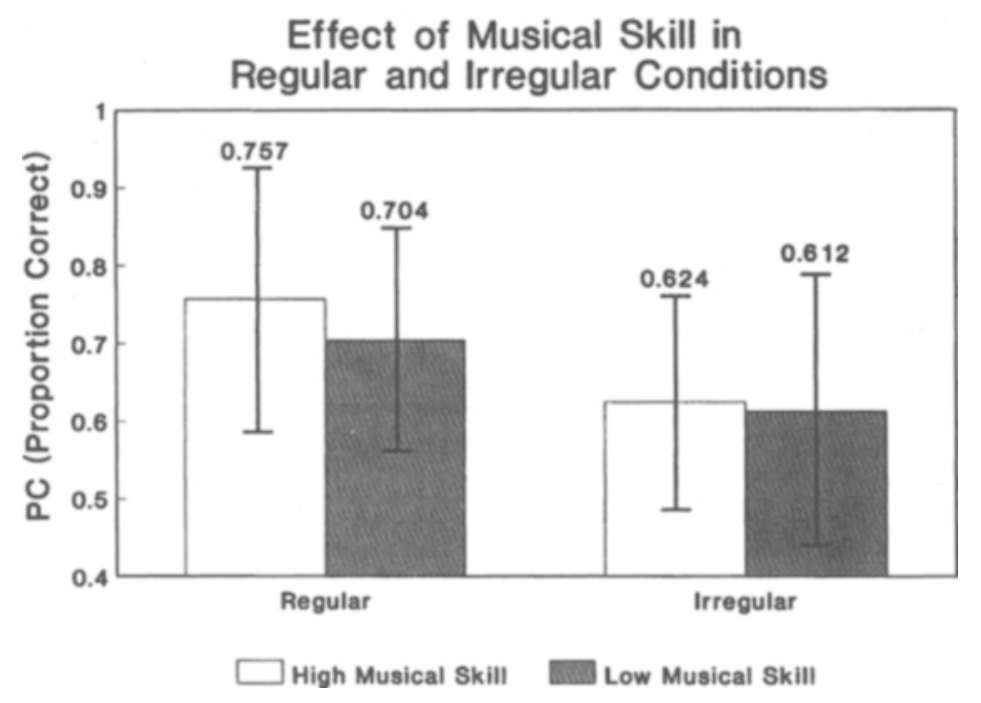

Figure 3. Mean proportion correct (PC) (also shown numerically) as a function of skill level and context in Experiment 2. Brackets indicate standard deviation per condition. 
Table 2

Proportion Correct Responses on Trial $\boldsymbol{n}$ Given Pattern Structure on Trial $n-1$ in Experiment 2

\begin{tabular}{lcccc}
\hline & \multicolumn{4}{c}{ Conditional Pattern Structure } \\
\cline { 2 - 5 } Skill Level & $\mathrm{R} / \mathrm{R}$ & $\mathrm{R} / \mathrm{I}$ & $\mathrm{I} / \mathrm{R}$ & $\mathrm{I} / \mathrm{I}$ \\
& $N=342$ & $N=738$ & $N=774$ & $N=270$ \\
\hline High & .645 & .731 & .577 & .571 \\
Low & .679 & .757 & .608 & .590 \\
\hline
\end{tabular}

Table 3

Proportion of Listeners Reporting Metrical and Anticipatory Attending in Experiment 2

\begin{tabular}{ccc}
\hline Skill Level & Metrical? & Anticipate? \\
\hline High & .72 & .78 \\
Low & .39 & .72 \\
\hline
\end{tabular}

Table 2 presents the probability of a correct response on a given pattern (regular, $\mathrm{R}$, or irregular, I) on Trial $n$, conditional on the type of sequence encountered on Trial $n-1$. These data are averaged over listeners within each skill level and trials $(N)$ to arrive at reliable point estimates. Not surprisingly, performance is better on $R$ than on $I$ patterns following both $I$ and $R$ sequences. However, the finding that the conditional probability of a correct response is highest when a regular pattern is preceded by an irregular one is unusual and not predicted by any of the models under consideration.

Finally, responses to the same questionnaires administered to subjects in Experiment 1 are summarized in Table 3. While tendencies to listen metrically are reported by high-skill listeners, these are not significantly above chance levels. The only reliable trend in these data (binomial tests) indicated that more high-skill listeners claimed to attend forward in time than they did backward in time $[Z=2.36, p<.01]$. However, chi-square analyses indicated no significant distributional differences as a function of skill level.

In general, these data do not replicate the findings of Experiment la. Instead, they show that the most important determinant of performance is the within-session manipulation of global pattern context (regular vs. irregular). In this respect, these data are difficult for Kristofferson's internal criterion model, but they replicate findings of Essens and Povel (1985), who reported systematic effects due to pattern context but not skill. Note, however, that this finding is the result of the elimination of skill differences found in Experiment $1 \mathrm{a}$ : high-skill listeners' performance in Experiment 2 drops dramatically relative to that in Experiment 1a. In this regard, the data are also consistent with the expectancy/ contrast model.

\section{GENERAL DISCUSSION}

In detecting small timing changes in simple acoustic sequences, musical skill aids listeners primarily when meaningful pattern structure can be exploited. Further- more, the situations that promote these effects involve global context. In particular, they relate to design constraints on the makeup of a session (within- vs. betweensubjects variables) and to general structural properties of individual patterns (regular vs. irregular).

The finding that design constraints affect overall accuracy levels and interactions among variables clarifies discordant findings in the literature regarding skill differences. Along with previous findings (Jones et al., 1981; Kidd et al., 1984; Mullenix, Pisoni, \& Martin, 1989), it underscores the importance of design choice. It appears that skill differences are more likely to appear in contexts where variability in structure is low.

Differences due to skill in the present task are not readily explained in terms of acuity differences, that is, smaller JNDs for skilled listeners. Skilled listeners were not significantly better than unskilled listeners with irregular sequences. Nevertheless, we do not wish to rule out the possibility that training refines temporal acuity, in part because high-skill listeners appeared to perform better than low-skill listeners on some irregular sequences in Experiment 1. The reasons for this are not clear, largely because the present designs, which randomly varied cycle durations of irregular patterns, do not permit decisive answers to such questions. Accordingly, a final judgment on the acuity hypothesis awaits orthogonal manipulations of both $\Delta t$ and $T$ for different skill groups in various carefully controlled pattern contexts.

Manipulation of pattern context constituted a major source of variance for all listeners in both experiments. The pattern of data as a function of skill speaks to the four hypotheses about determinants of $T$ mentioned in the introduction. Three models (average Weber, internal criterion, clock) deal with explicitly marked time intervals within a prescribed context, and a fourth admits implicitly marked time spans (the expectancy/contrast model). Of the first three, two fail to predict the present findings. The average Weber model, which proposes that $T$ depends on intervals immediately surrounding a time change, and the internal criterion model, which assumes that $T$ depends on the modal session IOI, opt for contexts that are defined either too narrowly (the average Weber model) or too broadly (the internal criterion model). Thus, the average Weber law model (Monahan \& Hirsh, 1990) incorrectly predicts null differences due to manipulations in pattern and session context because $\Delta t$ is gauged against a hypothetical $T$ value based on the average of the two explicitly marked test-cycle intervals, which are identical across these contexts. Generalizations of the averaging notion to global pattern context also fail to explain these data, because regular and irregular patterns are identical with respect to their average IOIs as well.

Kristofferson's internal criterion model, which assumes that the range of explicitly marked IOIs over a session determines performance, explains the fact that regular patterns are easier than irregular ones in Exper- 
iment la. However, it does not predict a skill $\times$ context interaction in that study, nor does it correctly predict pattern context effects in Experiment 2, in which the range and variance of IOIs within a session are identical for regular and irregular patterns. A modification of this model which assumes that the range of IOIs within a pattern determines performance also fails in that the range of IOIs within irregular patterns of Experiment 1a does not correlate significantly with detection accuracy.

Two other models, the clock model and the expectancy/contrast model, directly address pattern context. While Povel's clock fares poorly in Experiment 1, it receives clear support in Experiment 2. This requires further discussion.

According to Povel and Essens (1985), a listener's clock is based on recurrent time spans that are explicitly marked (accented) within a pattern. Against a variety of possible grids for the best fitting clock, the time interval that accumulates the least counterevidence throughout a sequence serves as the selected grid (i.e., the induced clock). We assume that this interval functions as $T$ in a time-detection task. It is important to observe that silence at the point of a hypothetical clock "tick" weighs heavily as counterevidence against that tick's marking out the time span of a selected clock. Thus, unless explicit markers (e.g., tone onsets) coincide with proposed clock "ticks," the corresponding interval is unlikely to be represented in the clock. Thus, as presently formulated, the clock model does not admit implied time spans based on subdividing silent intervals (Jones \& Yee, 1993). In short, $T$ cannot be an unmarked interval (e.g., $400 \mathrm{msec}$ ). We suggest that this contributes to the poor performance of this model in Experiment 1, where it does not predict an interaction of skill with pattern context. It implies that all listeners should ultimately arrive at the same best fitting explicitly marked time span (e.g., 1,600 or $800 \mathrm{msec}$ ).

Evidence that high-skill listeners in Experiment la may be relying on time intervals that are smaller than the explicitly marked ones of, for example, $800 \mathrm{msec}$, comes from several sources. In the first place, their performance with regular sequences having IOIs of $800 \mathrm{msec}$ was similar to that of low-skill listeners in Experiment $1 \mathrm{~b}$ who responded to regular sequences with marked IOIs of $400 \mathrm{msec}$. Secondly, questionnaire data suggest that listeners, especially high-skill listeners, engage in anticipatory attending and metrical listening to regular sequences. In addition, it was primarily in conditions where high-skill listeners were presented with regular sequences that spontaneous finger or toe tapping was observed during the experiment, an informal indication that these subjects were attempting to "find the beat." Finally, the data themselves converge nicely with those of Palmer and Krumhansl (1990), who found that listeners judged probe tones, variously located within larger sequences, to "fit" better into the prevailing context when they precisely subdivided a marked interval within the context (i.e., subdivided by 2 or 3 ). Here, too, high-skill listeners' judgments revealed reliance on a finer "temporal grain" than did low-skill listeners' judgments (see also Jones \& Yee, 1993).

The latter findings are consistent with the expectancy/ contrast approach, where expected time spans, ETs, including implied ones, can supply $T$ values. Figure 4 sug-

\section{FUTURE-ORIENTED ATTENDING and TEMPORAL CONTRAST}
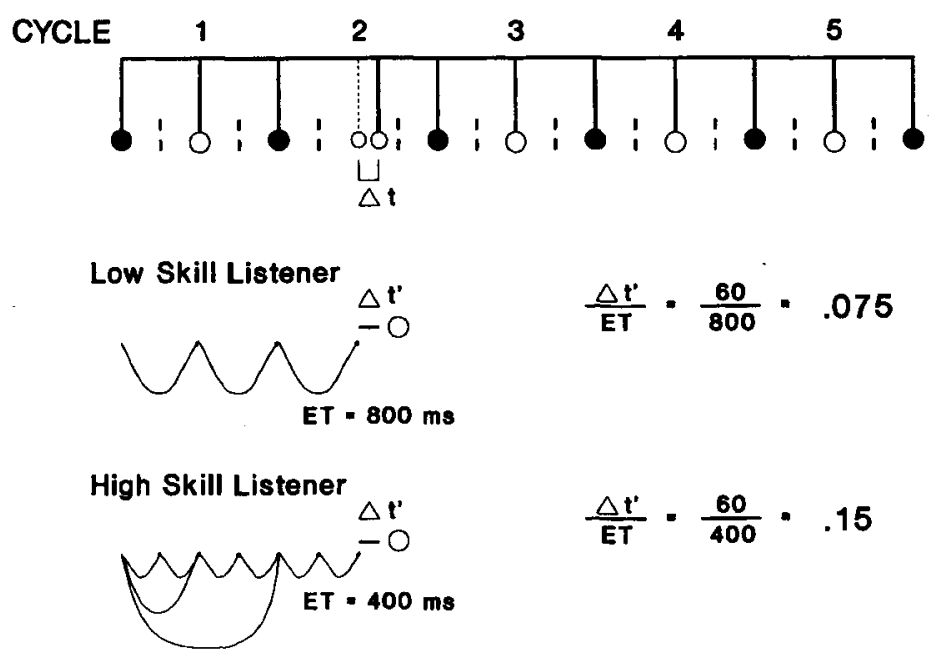

Figure 4. A schematic rationale for expectancy/contrast predictions regarding skill differences in regular sequences. Assuming that proportion correct is a function of $\Delta t^{\prime} / \mathrm{ET}$, high-skill listeners should be more accurate because they generate expected time-interval (ET) values corresponding to implied time spans of regular sequences. 
gests how these ideas apply to listeners who engage in future-oriented attending to regular sequences. Lowskill listeners base their expectancies on explicitly marked IOIs, whereas those with more training can infer implied time spans. More formally, Jones and Boltz (1989, Equation 2, p. 471) state that expectancies depend on two factors: (1) $T^{\prime}$, an estimate of the invariant base period (e.g., here IOI of $800 \mathrm{msec}$ ), and (2) $\mathrm{C}_{t}{ }^{\prime}$, an estimate of the invariant time ratio ${ }^{4}$ (e.g., here cycle duration/IOI $=2$ ). To illustrate, if $T^{\prime}$ approximates $800 \mathrm{msec}$ and $\mathrm{C}_{t}^{\prime}$ estimates 2 , then ET $=T^{\prime} \times \mathrm{C}_{t}^{n^{\prime}}$ specifies higher order periods of, for example, 1600 or 3,200 msec when $n=1$ and 2 , respectively. Smaller times spans correspond to negative values of $n$. Thus, the implied span of $400 \mathrm{msec}=800 \times 2^{-1}$ (for illustrative purposes only, we assume error-free estimates of parameters $T^{\prime}$ and $\left.\mathrm{C}_{t}^{\prime}\right)$. This view assumes that skill affects listeners' ability to iterate values of $n$ such that high-skill listeners are more likely to use $n \neq 0$, whereas low-skill listeners tend to rely on $n=0$. Thus, as shown in Figure 4, high-skill listeners can generate ETs in the neighborhood of $400 \mathrm{msec}$, whereas low-skill listeners will rely on ETs $=800 \mathrm{msec}$. We note that although the present account does not rule out the possibility of learning during a session (by either skilled or unskilled listeners), these predictions are not predicated on skilled listeners' acquiring a metrical listening skill within a particular experiment. Rather, as the data of Experiment 1 suggest, musical listeners are assumed to bring to the task a tendency to exploit values of $n$ in addition to $n=0$. Finally, when such expectancies are combined with the assumption that an expectancy violation, in the form of a temporal contrast, $\Delta t^{\prime}$, determines time-detection performance when gauged relative to the expected base period, ET (i.e., as in $\Delta t^{\prime} / \mathrm{ET}$ ), this model has some potential for describing the pattern of data observed in Experiment 1.

The time ratio (estimated by $\mathrm{C}_{t}^{\prime}$ ) in the above expression corresponds to the cycle/IOI subdivision, and this value is held constant in both experiments. However, variations in actual durational values of cycles are predicted to affect how reliably listeners can abstract $C_{t}$. Uniform entrainment to a stable time interval is a prerequisite to the abstraction and use of such ratio properties (Jones \& Boltz, 1989). Consequently, skilled listeners should have more difficulty with regular sequences in Experiment 2 than in Experiment 1, and they do. One interpretation of such findings is that the presence of higher order irregularities prevent musically skilled listeners from "finding an implied beat." Consistent with this is the finding that the number of high-skill listeners claiming to listen metrically in Experiment 1 was significantly above chance levels in Experiment 1 but not in Experiment 2. Nevertheless, it is clear that in Experiment 2 many high-skill listeners tried to listen metrically; indeed, a majority of listeners in both skill groups attempted to listen "ahead." However, relative to performance levels in Experiment 1, accuracy levels in Ex- periment 2 suggest that when listeners are denied a stable referent period, these strategies are not so effective even for trained listeners.

The present research was primarily designed to focus upon the way in which skilled and unskilled listeners respond to regular sequences. With irregular sequences, by contrast, it is clear that all listeners have difficulty detecting time changes. Detailed analyses of responses to these sequences suggest that different configurations of IOIs may tap the different listening strategies that people report. Limited attentional entrainments of analytic attending, based on local regularities within these sequences, may account for better performance on some of these patterns, but more controlled designs are necessary to sort out all of the contributing detvarious other contributing factors in responses to irregular sequences. At this point, these findings converge with recent findings reported by Handel (1992), who used different kinds of irregular sequences, to show that time discrimination is highly variable in irregular sequences.

\section{REFERENCES}

AвEL, S. M. (1972). Discrimination of temporal gaps. Journal of the Acoustical Society of America, 52, 519-524.

Allan, L. G. (1979). The perception of time. Perception \& Psychophysics, 26, 340-354.

AllaN, L. G., \& KRISTofferson, A. B. (1974). Judgements about the duration of brief stimuli. Perception \& Psychophysics, 15, 434-440.

Bharucha, J. J., \& Pryor, J. H. (1986). Disrupting the isochrony underlying rhythm: An asymmetry in discrimination. Perception \& Psychophysics, 40, 137-141.

Creflman, C. D. (1962). Human discrimination of auditory duration. Journal of the Acoustical Society of America, 34, 582-593.

EsSENS, P. J. (1986). Hierarchical organization of temporal patterns. Perception \& Psychophysics, 40, 60-73.

ESSENS, P. J., \& Povel, D. J. (1985). Metrical and nonmetrical representations of temporal patterns. Perception \& Psychophysics, 37, 1-7.

GETTY, D. J. (1975). Discrimination of short temporal intervals: A comparison of two models. Perception \& Psychophysics, 18, 1-8.

HALPERN, A. R., \& DARWIN, C. J. (1984). Duration discrimination in a series of rhythmic events. Perception \& Psychophysics, 31, 86-89.

HANDEL, S. (1992). The differentiation of rhythmic structure. Perception \& Psychophysics, 52, 497-507.

Hirsh, I. J., Monahan, C. B., Grant, K. W., \& Singh, P. G. (1990). Studies in auditory timing: 1. Simple patterns. Perception \& Psychophysics, 47, 215-226.

JoNEs, M. R. (1976). Time, our lost dimension: Toward a new theory of perception, attention, and memory. Psychological Review, 83, 323-335

Jones, M. R., \& Boltz, M. (1989). Dynamic attending and responses to time. Psychological Review, 96, 459-491.

Jones, M. R., BoltZ, M., \& KLEIN, M. (1993). Expected endings and judged duration. Memory \& Cognition, 21, 646-665.

Jones, M. R., JAGACINSKI, R. J., YEE, W., FlOYD, R. L., \& KLAPP, S. T. (in press). Tests of attentional flexibility in listening to polyrhythmic patterns. Journal of Experimental Psychology: Human Perception \& Performance.

JONES, M. R., KIDD, G., \& WETZEL, R. (1981). Evidence for rhythmic attention. Journal of Experimental Psychology: Human Perception \& Performance, 7, 1059-1073.

JONES, M. R., \& YEE, W. (1993). Attending to auditory events: The role of temporal organization. In S. McAdams \& E. Bigand (Eds.), Thinking in sound: The cognitive psychology of human audition (pp. 69-112). Oxford: Oxford University Press. 
KIDD, G., Boltz, M., \& Jones, M. R. (1984). Some effects of rhythmic context melody recognition. American Journal of Psychology, 97, 153-173.

KiDD, G. R., \& WaTSON, C. S. (1990). Detection of relative-duration changes in tonal patterns. Journal of the Acoustical Society of America, 86, S121. (Abstract)

Kristofferson, A. B. (1976). Low-variance stimulus-response latencies: Deterministic internal delays? Perception \& Psychophysics, 20, 89-100.

KrISTOFFERSON, A. B. (1977). A real-time criterion theory of duration discrimination. Perception \& Psychophysics, 21, 105-117.

Monahan, C. B., \& Hirsh, I. J. (1990). Studies in auditory timing: 2. Rhythm patterns. Perception \& Psychophysics, 47, 227-242.

Mullenix, J. W., Pisoni, D. B., \& Martin, C. S. (1989). Some effects of variability on spoken word recognition. Journal of the Acoustical Society of America, 85, 365-378.

Palmer, C., \& Krumhansl, C. L. (1990). Mental representations for musical meter. Journal of Experimental Psychology: Human Perception \& Performance, 16, 728-741.

Povel, D. J., \& Essens, P. J. (1985). Perception of temporal patterns. Music Perception, 2, 411-440.

SLOBODA, J. A. (1983). The communication of musical in piano performance. Quarterly Journal of Experimental Psychology, 35A, 377-390.

Small, A. M., \& Campbell, R. A. (1962). Temporal differential sensitivity for auditory stimuli. American Journal of Psychology, 75, 401-410.

Thomas, E. A., \& Brown, I., JR. (1974). Time perception and the filled duration illusion. Perception \& Psychophysics, 16, 449-458.

Todd, R. E., Boltz, M., \& JonEs, M. R. (1989). The MIDILAB music research system, Psychomusicology, 8, 17-30.

TUKEY, J. W. (1977). Exploratory data analysis. Reading, MA: Addison-Wesley.

VAN NOORDEN, L. P. A. S. (1975). Temporal coherence in the percep- tion of tone sequences. Unpublished doctoral dissertation, Eindhoven University of Technology, The Netherlands.

\section{NOTES}

1. Functionally speaking, an "accent" is any attention-getting element of a sequence. Typically, accents correspond to significant serial changes along one or more dimensions of physical structure within the sequence (e.g., intensity, frequency, time). Thus, louder sounds are accented elements in the clock model, and they have priority in defining the "best fitting" grid. Povel and Essens (1985) developed a formula based on counterevidence (see General Discussion) to quantify "best fit"; the formula negatively weights alignments of clock "ticks" to pattern elements such as unaccented elements and silences.

2. Temporal contrast defined strictly as OT - ET is oversimplified when ET realizes generated time spans and OT is taken merely as an IOI. One or the other must be adjusted to capture the notion that the two spans are supposed to initiate at the same onset time. For example, if OT is IOI $+\Delta t$ (e.g., $860 \mathrm{msec})$ and the expected point in time is based on a smaller implied span (e.g., ET $=400 \mathrm{msec}$ ), then contrast is really based on the consequence of a series of beats (ETs) within IOI: $\Delta t^{\prime}=\mathrm{OT}-2 \mathrm{ET}$. The more general expression is $\Delta t^{\prime}=(\mathrm{OT}-n \mathrm{ET})$.

3. This analysis was performed at the suggestion of Steve Handel.

4. Equation 2 (Jones \& Boltz, 1989) is: $T_{n}^{\prime}=T_{j}^{\prime} \mathrm{C}_{t}^{\prime n-j}$ where $\mathrm{C}_{t}^{\prime}$ is the abstracted time ratio (here $\mathrm{C}_{t}^{\prime}=2$ ),$T_{n}^{\prime}$ is the expected time span, ET (here, e.g., $400 \mathrm{msec}$ ), $T_{j}^{\prime}$ is the referent or base time interval (here, e.g., $\mathrm{IOI}=800 \mathrm{msec}$ ), and the exponent $n-j$ represents generative interaction associated with relationships between levels $n$ (here $n=1$ ) and $j$ (here $j=2$ ). Thus, $T_{n}^{\prime}=800 \times 2^{-1}$, and hence, ET $=T_{n}^{\prime}=$ $400 \mathrm{msec}$.

(Manuscript received August 20, 1993; revision accepted for publication April 11, 1994.) 